\title{
A citizen science approach to evaluating US cities for biotic homogenization
}

\author{
Misha Leong ${ }^{\text {Corresp., } 1}$, Michelle D Trautwein ${ }^{1}$ \\ ${ }^{1}$ California Academy of Sciences, Institute of Biodiversity Science and Sustainability, San Francisco, California, United States of America \\ Corresponding Author: Misha Leong \\ Email address: mleong@calacademy.org
}

Cities around the world have converged on structural and environmental characteristics that exert similar eco-evolutionary pressures on local communities. However, evaluating how urban biodiversity responds to urban intensification remains poorly understood because of the challenges in capturing the diversity of a range of taxa within and across multiple cities from different types of urbanization. Here we utilize a growing resource-citizen science data. We analyzed 66,209 observations representing 5,209 species generated by the City Nature Challenge project on the iNaturalist platform, in conjunction with remote sensing (NLCD2011) environmental data, to test for urban biotic homogenization at increasing levels of urban intensity across 14 metropolitan cities in the United States. Based on community composition analyses, we found that while similarities occur to an extent, urban biodiversity is often much more a reflection of the taxa living locally in a region. At the same time, the communities found in high intensity development were less explained by regional context than communities from other land cover types were. We also found that the most commonly observed species are often shared between cities and are non-endemic and/or have a distribution facilitated by humans. This study highlights the value of citizen science data in answering questions in urban ecology. 


\section{A citizen science approach to evaluating US cities for 2 biotic homogenization}

3

4

Misha Leong and Michelle Trautwein

Institute for Biodiversity Science and Sustainability, California Academy of Sciences, San Francisco CA, United States

Corresponding Author:

Misha Leong

55 Music Concourse Drive, San Francisco, CA

Email address: MLeong@calacademy.org

\section{Abstract}

Cities around the world have converged on structural and environmental characteristics that exert similar eco-evolutionary pressures on local communities. However, evaluating how urban biodiversity responds to urban intensification remains poorly understood because of the challenges in capturing the diversity of a range of taxa within and across multiple cities from different types of urbanization. Here we utilize a growing resource-citizen science data. We analyzed 66,209 observations representing 5,209 species generated by the City Nature Challenge project on the iNaturalist platform, in conjunction with remote sensing (NLCD2011) environmental data, to test for urban biotic homogenization at increasing levels of urban intensity across 14 metropolitan cities in the United States. Based on community composition analyses, we found that while similarities occur to an extent, urban biodiversity is often much more a reflection of the taxa living locally in a region. At the same time, the communities found in high intensity development were less explained by regional context than communities from other land cover types were. We also found that the most commonly observed species are often shared between cities and are non-endemic and/or have a distribution facilitated by humans. This study highlights the value of citizen science data in answering questions in urban ecology. 
34

35

36

37

38

39

40

41

42

43

44

45

46

47

48

49

50

51

52

53

54

55

56

57

58

59

60

61

62

63

64

65

66

67

68

69

70

71

72

73

\section{Introduction}

Cities around the world exist in a range of environmental contexts, yet because of the requirements and preferences of their human inhabitants, they share commonalities such as landscape fragmentation, altered water and resource availability, and high densities of fabricated structures and impervious surfaces that alter climate (Rebele, 1994). With this ecological homogenization (Groffman et al., 2014) come potential consequences on the biodiversity of the organisms that live in and around cities (Savard, Clergeau \& Mennechez, 2000). Plants have been found to bloom earlier in city centers due to the urban heat island effect (Mimet et al., 2009), bird migratory patterns have shifted to take advantage of resource availability (Tryjanowski et al., 2013), and invasive species can be more prominent because of increased rates of species introductions (Tsutsui et al., 2000). While such modifications are still relatively recent on an evolutionary time scale, phenotypic changes have been observed across taxa on a global scale as eco-evolutionary consequences of urbanization (Alberti, 2015). Understanding such changes can help us better plan for future ecological dynamics in cities, such as predicting population vulnerability to invasive species or minimizing human-wildlife conflicts, such as property damage or health hazards (eg. disease vectors).

Common ecological metrics such as species richness and abundance have shown mixed results in urban environments. A review of 105 studies on species richness along urban to rural gradients demonstrated inconsistent patterns - while some studies found that species richness decreases with higher urban intensification, other studies found the opposite (McKinney, 2008). Often, this greater than expected species richness can be largely attributed to non-native species (McKinney, 2008), highlighting the importance of additionally considering shifts in community composition. The commonality and spread of urban specialists could contribute to urban biotic homogenization - the idea that on a global scale the biodiversity of cities converges (McKinney, 2006; La Sorte, McKinney \& Pyšek, 2007; Clavel, Julliard \& Devictor, 2011). This has been particularly observed to occur with urban plants (Schwartz, Thorne \& Viers, 2006; Pearse et al., 2018), and driven concerns on the cascading impacts reductions in beta diversity could have for conservation (Socolar et al., 2016).

A challenging aspect to measuring urban homogenization is gathering sufficient data to cover the variation in ecological communities within and between cities. Within city biodiversity levels can vary greatly by neighborhood (Sushinsky et al., 2013). To address this, cities have frequently been examined along rural to urban gradients, although this method has been criticized for its oversimplification of features and the vagueness of definitions that makes comparisons between cities difficult (McDonnell \& Hahs, 2008). Broad terminology like "urban" can refer to dense downtown built-up environments, residential neighborhoods, industrial areas, or parks. Even within a single type, such as residential neighborhoods, factors such as socioeconomic demographics or landscape legacy can contribute to even more local habitat heterogeneity (Leong, Dunn \& Trautwein, 2018).

One solution to capturing all this variation and exploring patterns of biodiversity across geographically disparate cities is to utilize data generated through public engagement. Broadly 
74 referred to as citizen science (although we emphasize that one need not be a citizen of any

75

76

77

78

79

80

81

82

83

84

85

86

87

88

89

90

91

92

93

94

95

96

97

98

99

100

101

102

103

104

105

106

107

108

109

110

111

112

nationality to participate), this process involves public collaboration with professional scientists in ways that help our understanding of the natural world (Ballard et al., 2017). Citizen science data collection overcomes the challenges of accessing private land and can be scaled up to cover multiple cities with relative ease (Spear, Pauly \& Kaiser, 2017). There are obvious challenges such as collection biases and identification quality that need to be accounted for (Isaac et al., 2014), but citizen science is a potentially valuable tool that can be used far beyond science engagement or exploring expanding species distributions.

Here we examine patterns in urban biodiversity across 14 metropolitan areas in the United States using data generated by the general public. We take a multi-scale approach to examine urban biotic homogenization both between and within cities. Specifically, we ask 1) how biodiversity is shared between cities across different regions; and 2) whether the effect of biotic homogenization gets stronger as urbanization intensifies.

\section{Materials \& Methods}

The City Nature Challenge is a citizen science initiative started by the California Academy of Sciences and the Los Angeles Museum of Natural History that utilizes the iNaturalist platform to encourage users to photograph urban nature during a bioblitz in late April. For the 16 cities that participated in 2017 (San Francisco CA, Los Angeles CA, Seattle WA, Salt Lake City UT, Austin TX, Houston TX, Dallas TX, Duluth, MN, Minneapolis MN, Chicago IL, Nashville TN, Miami FL, Raleigh NC, Washington DC, New York NY, and Boston MS) we accessed all available City Nature Challenge data from for all years available. Next, we filtered all observations to include "Research Grade" only, which is defined by the iNaturalist platform as being verifiable with a photograph and having reached a species identification consensus by at least 2 users in the iNaturalist community (more details available at inaturalist.org). We further filtered these observations to only include those observations that had open and un-obscured geocoordinates (geoprivacy both by user choice and for species with a conservation status are maintained on the iNaturalist platform). Because this reduced the number of available observations, we excluded the cities of Duluth and Nashville from further analyses. The 14 included metropolitan areas (Fig. 1) cover a range of geographic and environmental diversity. There were a range of number of observations between cities, highlighting the disproportionate sampling effort, with Miami having the fewest observations at 1,011 and the San Francisco Bay Area having the most at 15,733. The average number of observations of the 14 cities was 5,077 $+/-3817$. Differences in collecting effort are addressed in our analyses by using techniques such as within city comparisons and community composition metrics.

All data and scripts used for the following analyses can be found at https://github.com/mishoptera/cnc.

\section{Shared biodiversity between cities}


113

114

115

116

117

118

119

120

121

122

123

124

125

126

127

128

129

130

131

132

133

134

135

136

137

138

139

140

141

142

143

144

145

146

147

148

149

150

151

152

We identified which species were found in the majority of the cities to compare these widespread species with the total pool of observations. We also divided the dataset by major taxa: 4 plant groups (monocots, dicots, ferns, and conifers) and 6 animal groups (birds, insects, reptiles, amphibians, mammals, and gastropods), such as to allow for better comparisons between similar taxa. To capture observed species from groups that had insufficient observations on their own (e.g. isopods, fungi, arachnids), we also created a catch-all "other" category.

\section{Biotic homogenization with increasing urban intensification}

After seeing how biodiversity was shared between cities, we asked whether the biotic homogenization effect was stronger with increasing urbanization intensity. Based on geographic coordinates, we linked all observations with a NLCD2011 land cover classification from the Multi-Resolution Land Characteristics Consortium (MRLC). Assessed nationwide at a 30 x 30m resolution, every pixel is assigned one of 16 land cover classifications, four of which are forms of developed land with increasing urbanization intensity (developed-open space, developed-low intensity, developed-medium intensity, developed-high intensity; further details in Table 1). We collapsed the remaining land cover classifications into "water", "agricultural", and "natural". As we were only interested in comparing increasing levels of urbanization against the natural land use type, we excluded any observations that were classified as having occurred within agricultural or water pixels.

We then analyzed the relative influence of level of urban intensification and city on community composition. To do this, we built Bray-Curtis dissimilarity matrices based on the species composition at each level of urbanization within each city, and visualized community composition using NMDS (Non-Metric multi-Dimensional Scaling) with 100 restarts. We applied a stress cut-off of 0.20 ; if stress was $>0.20$, we considered the NMDS plot to be unreliable (Quinn \& Keough, 2002). We visualized groupings both based on land cover type and by city.

As regional location can be an important environmental filter in determining community composition (Williams et al., 2009; Aronson et al., 2014; Pearse et al., 2018), we also created NMDS plots for regional groups in a series of triads of increasing geographic distance. Specifically, we focused on a Texas group (Houston, Dallas, and Austin), Atlantic Coast group (New York City, Boston, and Washington DC), Pacific Coast group (Seattle, Los Angeles, and San Francisco), and a fairly widespread Central United States group (Salt Lake City, Minneapolis, and Chicago) (Fig. 1).

To examine whether community composition becomes more similar with increased urban intensification, we subdivided observations based on their land cover classification (natural, developed-open space, developed-low intensity, developed-medium intensity, developed-high intensity). We then looked for the effect of regional location (with three city "replicates" for each region as above - Raleigh and Miami were excluded from this analysis because they did not fall neatly into one of the other regional categories). We built a PERMANOVA (Permutational Multivariate Analysis of Variance, (Anderson, 2017)) model for each land cover group with 999 
153 iterations based on Bray-Curtis dissimilarity (R package vegan, (Oksanen et al., 2015)), then

154 compared the $\mathrm{R}^{2}$, $\mathrm{p}$-value and AIC score for each of the models generated by the five different

155 land cover classifications. We would expect that if biotic homogenization were occurring with

156 increased urban intensification, the models built off of the observations from the more developed

157 land cover types would perform less well because the effect of regional location should be

158 reduced.

159

160

\section{Results and Discussion}

161

162

163

164

165

166

167

168

169

170

171

172

173

174

175

176

177

178

179

180

181

182

183

184

185

186

187

188

189

190

191

192

\section{Shared biodiversity between cities}

We analyzed 66,209 citizen science research grade iNaturalist observations across 14 US metropolitan areas. Overall, dicots, the largest plant group, were overwhelmingly the most observed (59.6\%) and had the most species (52.4\%). The next most observed groups were birds $(12.8 \%)$, monocots $(8.7 \%)$, and insects $(8 \%)$. However, despite making up only $8 \%$ of the observations, insects actually made up $18.4 \%$ of the total species richness. Birds, on the other hand, made up only $7.8 \%$ of species richness, meaning they have a higher proportion of number of observations per species.

Of the 5,209 observed species, exactly 100 were found in the majority ( 8 or more) of the cities (Table 2), which we hereafter refer to as our "cosmopolitan" species. While the cosmopolitan species were primarily birds and dicots (36 each), and a few mammals (7), insects (7), and reptiles (4), there was also one cosmopolitan species each for amphibians, monocots, and conifers, and no representative species for gastropods or ferns. Although only $1.9 \%$ of the total species richness, these widespread cosmopolitan species made up $21.4 \%$ of the total observations. Two birds, the rock dove and American crow, were the only species observed in each of the 14 cities. Ten additional species were observed in 13 cities each -7 of which were also birds (red-winged blackbird, mallard, great blue heron, turkey vulture, house sparrow, American robin, and mourning dove), but also one dicot (common dandelion), one insect (Asian lady beetle), and one mammal (common raccoon).

Taxa varied in how cosmopolitan (again, here defined as being found in the majority of our cities) they were as a group. Mammals and birds had the highest proportions of cosmopolitan species (10.6\% and $10.1 \%$ respectively). On the opposite end of the spectrum, insects and dicots had a much smaller proportion of their species observed in the majority of cities $(0.83 \%$ and $1.5 \%$ respectively). Our findings that cities comprise a few cosmopolitan species with a mix of many local species complement other findings that the majority of urban species are still local species (Aronson et al., 2014).

However, these cosmopolitan species accounted for the majority of observations for mammals (55.2\%) and birds (64.8\%), and even made up a large proportion of observations for insects $(25.3 \%)$ and dicots $(15.7 \%)$. While it is possible that these patterns could also be explained by cosmopolitan species being more recognizable to people (and therefore more frequently identified, leading to an inflation in the proportion of observations for these groups),

Peer) reviewing PDF | (2019:01:34115:1:0:NEW 19 Mar 2019) 
193 the substantial proportion of cosmopolitan species could also be indicative of a downward trend 194 of the relative abundance of native species populations in cities. Previous multi-city studies of

195 biotic homogenization have relied on species lists (Aronson et al., 2014), which can not capture 196 shifts in community proportions. With mass species declines in tropical and temperate 197 ecosystems (Hallmann et al., 2017; Lister \& Garcia, 2018), such findings of cosmopolitan 198 species making up such a large portion of the community relative to native species merit further 199 investigation.

200

201

202

203

204

205

206

207

208

209

210

211

212

213

214

215

216

217

218

219

220

221

222

223

224

225

226

227

228

229

230

231

232

\section{Biotic homogenization with increasing urban intensification}

We next asked whether the effect of biotic homogenization grows stronger as a landscape becomes more developed through urbanization. The clustering in our NMDS plots suggest that urban biodiversity is to some degree city specific but also tied to particular levels of urbanization (Fig. 2). Plants exhibited a slightly different pattern from animals (Fig. S1), with the plant communities observed in the highest levels of urban intensification having the greatest differentiation, opposite to the pattern that would be expected if urban homogenization were occurring. This contrasts with a previous study that found that across cities, cultivated yards tended to be more similar to one another compared to the similarity of their associated natural areas across cities (Pearse et al., 2018), which could be due to being unable to differentiate between cultivated and spontaneous vegetative growth observations, and the iNaturalist platform discourages the recording of cultivated plants and animals.

We found that communities, regardless of level of urban intensification, within the same city were found close together on the NMDS plots - a pattern further reinforced by region (Fig. 2b). For example, all three Texan metropolitan cities (Houston, Dallas, and Austin) were grouped near one another, as were the cities along the Atlantic (Boston, New York City, and Washington DC) and Pacific Coasts (Seattle, San Francisco, and Los Angeles). Miami, being more geographically isolated and environmentally distinct than the other cities was relatively far on the plot from the other cities. Such findings complement what we found on the between cities comparison, where urban communities are largely a reflection of the local regional community, with a few cosmopolitan species. This regional clustering was found for both plants and animals. Animal communities overall were more similar between cities than plant communities, perhaps because of their mobility and ability to respond relatively quickly to land cover changes.

In the regional triad NMDS plots (Fig. 3) which peeled away some of the environmental variation between cities, community composition showed overlap between the different levels of urbanization in an ordered way along the urbanization spectrum, in that more similar levels of urbanization also share more similar communities. In all four regional groups, community composition from high intensity urbanization were more distinct than those from all other land cover types - even more distinct than those from natural were from the least developed areas. For the Atlantic and Pacific Coast cities, there appeared to be a longitudinal gradient, with the cities falling in the geographic middle (New York City and San Francisco respectively) having all of their land cover community compositions falling between the community compositions of 
233 cities that were more north and south. The distinctness of communities from each land cover type 234 were more evident in those triads that have cities that are geographically closer to one another. In 235 other words, as environmental context becomes less variable, levels of urbanization become

236

237

238

239

240

241

242

243

244

245

246

247

248

249

250

251

252

253

254

255

256

257

258

259

260

261

262

263

264

265

266

267

268

269

270

271

272 more important in defining the community composition.

As predicted, the PERMANOVA models (for all observations, plants only, and animals only) built from observations from high intensity land cover performed the poorest (Table 3), meaning that regional group membership was less able to predict community composition in higher intensity land cover than it could in the other land cover types. This is consistent with what we would expect to occur if biotic homogenization increases with urbanization intensification. However, the effect of regional group is still significant $(p<0.001)$ even for high intensity land cover observations. Additionally, while the PERMANOVA models built from the high intensity land cover observations appear the weakest (based on AIC and $\mathrm{R}^{2}$ ), the models based on observations from natural and the other developed land cover types did not appear to decrease in strength in an ordered way with increasing urban intensification.

\section{Additional observations}

Many species demonstrated a preferential association for either natural or high-intensity urban areas across all the cities they were found in. In general, we found that those species that favored higher intensity urban land cover tended to be non-natives, having origins in Europe, North Africa, and South Africa (e.g. common dandelion, white clover, common ivy, house sparrow, rock dove, common starling). Conversely (and expectedly), those that were found to favor more natural sites are native to North America (e.g. poison ivy, Virginia creeper, northern cardinal). However, it was difficult to identify specific ecological traits that urban specialists shared, as has been a similar finding in other urban ecology studies (Duncan et al., 2011)

Among the widespread cosmopolitan species we identified in the between cities comparison, we expected there to be a preferential association for the higher intensity land use types. There were in fact several species that showed this pattern-such as the house sparrow and rock dove. However, just as many widespread species favored the less disturbed natural land cover types - such as the white tail deer. It seems there are multiple human-associated mechanisms that act at different scales. Human transportation networks, as well as agriculture and other human directed habitat shifts have facilitated species introductions and expanded species ranges, while urbanization has created unique habitats that allow particular species to thrive. While humans are a common denominator, species that benefit from range expansions do not necessarily also benefit from urbanization.

The western honey bee is an example of a species that varied greatly in which land cover type it favored - it was most frequently observed in the highest intensity urban land cover types in Washington DC and Los Angeles, the natural land cover types for Austin, and somewhere along the urbanization spectrum for everywhere else. The honey bee was found in every city except Minneapolis and Seattle, and was most frequently observed in cities in Texas and California. Pollinators, and honey bees in particular, have been shown to be sensitive to climatic 
273 differences (Gordo \& Sanz, 2006; Bartomeus et al., 2011), and the varying environmental 274 conditions between cities in April could explain why the honey bee was not found in the two 275 northernmost cities and most abundant in the more southern ones. Further, the "snapshot" 276 approach of the City Nature Challenge captures cities at different points in their seasonal 277 progression, as bee abundance phenology is known to vary between land cover types (Leong et 278 al., 2016).

279 Many frequently observed species are also invasive species - such as garlic mustard. 280 While originally introduced to North America from Europe, it thrives in the forest understory

281

282

283

284

285

286

287

288

289

290

291

292

293

294

295

296

297

298

299

300

301

302

303

304

305

306

307

308

309

310

311 (Stinson et al., 2006). It was particularly abundant in Boston, New York, and Washington D.C., where it was found across all land cover types. Because there are many ongoing efforts to control this species (Nuzzo, 1999; Blossey et al., 2001), it will be important for land managers to consider that urban landscapes could also act as reservoirs maintaining sizeable populations of this species.

Our methodology utilizes within city and land cover type community composition and ranking metrics to avoid biases based on "collecting effort." However, there remain other challenges in teasing apart patterns reflecting ecological dynamics and natural history versus artifacts associated with data collected opportunistically by members of the public that currently limit ways in which we can interpret our findings. For example, species with the most observations are often not truly the most abundant species in cities, rather they are the easiest to photograph and identify (hence, the "overrepresentation" of bird taxa). Insects and other small taxa that are more difficult to photograph and identify are almost certainly under recorded. Many species were rarely observed - 2435 of the 5,209 total species included in the dataset were singleton/doubletons, meaning they were only observed once or twice. Although we can assume that most species should be relatively equally photographable and identifiable across land cover types, we recommend using multiple approaches to make comparisons "within the biases," such as focusing on community composition and nonparametric statistical methods as we have done here.

\section{Conclusions}

Our findings provide some support for biotic homogenization, although no single species was recorded in the highest level of urbanization across all cities. While we find that community composition is significantly impacted by degree of urban intensification, the role of geographic and environmental region seems to have a larger role in determining communities. Urban biodiversity is a mix of local natural biodiversity and introduced species that are closely associated with humans. These novel "hybrid ecosystems," with both local regional filters and the human influences of dispersal and resources are a growing reality in many parts of the world, and are continually changing with species adapting to exploit them (Kowarik, 2011). While it has been suggested that cities can act as reservoirs for native biodiversity (Pearse et al., 2018), 
312 conversely, natural areas can also be impacted by the diversity of species in the cities that they 313 border.

314 Despite the complexity of urban biodiversity dynamics, this work demonstrates the power 315 of using citizen science data in urban landscapes. The data from the City Nature Challenge 316 provide an opportunity to look at diverse species occurrences across many cities during the same 317 snapshot of time in a manner that has not been possible before. The opportunistic nature of 318 citizen science data is comparable to natural history collections in many ways (Spear, Pauly \& 319 Kaiser, 2017), yet with an additional factor of being focused in urban landscapes. Further, citizen 320 science data makes up a large proportion of GBIF data and is continuing to grow at a fast rate. 321 There are many potential future questions to explore, particularly as this dataset continues to 322 grow in conjunction with other large environmental datasets.

323 While we focused our efforts using a subset of available iNaturalist observation data from 324 the City Nature Challenge and the levels of urbanization from the National Land Cover 325 Database, there are many more environmental and geopolitical datasets available that can be 326 used to explore patterns in urban biodiversity. Expanding our scope to include all iNaturalist 327 observations and museum collection specimen data could help untangle some of the complexity 328 that we observed. Future work can also pursue broader ecological questions such as the role of 329 climate change on urban biodiversity, phenological shifts, city connectedness, links with 330 socioeconomics, the historical legacies of cities, and how these patterns change over time.

Finally, beyond the value that citizen science data can provide in allowing us to ask questions that would have been impossible to previously explore, the collection of these data engages the broader public in the ecological and environmental world around them in a meaningful way. An engaged network of citizen scientists is a built-in audience for science communication, making citizen science a valuable tool to increase the relevancy of environmental research. The everyday biodiversity in cities is now known to be an important contributor to city resident well-being and health. Concerns about the growing disconnect between city residents and nature can be combated (Schuttler et al., 2018) with increased awareness and participation in decision-making to build healthier and happier cities.

\section{Acknowledgements}

We thank all organizers and participants of the City Nature Challenge. In particular, we are grateful to Alison Young (California Academy of Sciences) and Lila Higgins (Los Angeles Natural History Museum) as the co-founders and lead global organizers of CNC, and Amy Jaecker-Jones as the global coordinator of CNC.

\section{References}


351

352

353

354

355

356

357

358

359

360

361

362

363

364

365

366

367

368

369

370

371

372

373

374

375

376

377

378

379

380

381

382

383

384

385

386

387

388

389

390

391

392

393

394

395

396
Alberti M. 2015. Eco-evolutionary dynamics in an urbanizing planet. Trends in Ecology \& Evolution 30:114-126. DOI: 10.1016/j.tree.2014.11.007.

Anderson MJ. 2017. Permutational Multivariate Analysis of Variance (PERMANOVA). In: Wiley StatsRef: Statistics Reference Online. American Cancer Society, 1-15. DOI: 10.1002/9781118445112.stat07841.

Aronson MFJ, Sorte FAL, Nilon CH, Katti M, Goddard MA, Lepczyk CA, Warren PS, Williams NSG, Cilliers S, Clarkson B, Dobbs C, Dolan R, Hedblom M, Klotz S, Kooijmans JL, Kühn I, MacGregor-Fors I, McDonnell M, Mörtberg U, Pyšek P, Siebert S, Sushinsky J, Werner P, Winter M. 2014. A global analysis of the impacts of urbanization on bird and plant diversity reveals key anthropogenic drivers. Proc. R. Soc. B 281:20133330. DOI: 10.1098/rspb.2013.3330.

Ballard HL, Robinson LD, Young AN, Pauly GB, Higgins LM, Johnson RF, Tweddle JC. 2017. Contributions to conservation outcomes by natural history museum-led citizen science: Examining evidence and next steps. Biological Conservation 208:87-97. DOI: 10.1016/j.biocon.2016.08.040.

Bartomeus I, Ascher JS, Wagner D, Danforth BN, Colla S, Kornbluth S, Winfree R. 2011. Climate-associated phenological advances in bee pollinators and bee-pollinated plants. Proceedings of the National Academy of Sciences 108:20645-20649. DOI: 10.1073/pnas.1115559108.

Blossey B, Nuzzo V, Hinz H, Gerber E. 2001. Developing biological control of Alliaria petiolata (M. Bieb.) Cavara and Grande (Garlic Mustard). Natural Areas Journal 21:357-367.

Clavel J, Julliard R, Devictor V. 2011. Worldwide decline of specialist species: toward a global functional homogenization? Frontiers in Ecology and the Environment 9:222-228. DOI: $10.1890 / 080216$.

Duncan RP, Clemants SE, Corlett RT, Hahs AK, McCarthy MA, McDonnell MJ, Schwartz MW, Thompson K, Vesk PA, Williams NSG. 2011. Plant traits and extinction in urban areas: a meta-analysis of 11 cities. Global Ecology and Biogeography 20:509-519. DOI: 10.1111/j.1466-8238.2010.00633.x.

Gordo O, Sanz JJ. 2006. Temporal trends in phenology of the honey bee Apis mellifera (L.) and the small white Pieris rapae (L.) in the Iberian Peninsula (1952-2004). Ecological Entomology 31:261-268. DOI: 10.1111/j.1365-2311.2006.00787.x.

Groffman PM, Cavender-Bares J, Bettez ND, Grove JM, Hall SJ, Heffernan JB, Hobbie SE, Larson KL, Morse JL, Neill C, Nelson K, O’Neil-Dunne J, Ogden L, Pataki DE, Polsky C, Chowdhury RR, Steele MK. 2014. Ecological homogenization of urban USA. Frontiers in Ecology and the Environment 12:74-81. DOI: 10.1890/120374.

Hallmann CA, Sorg M, Jongejans E, Siepel H, Hofland N, Schwan H, Stenmans W, Müller A, Sumser H, Hörren T, Goulson D, Kroon H de. 2017. More than 75 percent decline over 27 years in total flying insect biomass in protected areas. PLOS ONE 12:e0185809. DOI: 10.1371/journal.pone.0185809.

Isaac NJB, Strien AJ van, August TA, Zeeuw MP de, Roy DB. 2014. Statistics for citizen science: extracting signals of change from noisy ecological data. Methods in Ecology and Evolution 5:1052-1060. DOI: 10.1111/2041-210X.12254.

Kowarik I. 2011. Novel urban ecosystems, biodiversity, and conservation. Environmental Pollution (Barking, Essex: 1987) 159:1974-1983. DOI: 10.1016/j.envpol.2011.02.022.

Leong M, Dunn RR, Trautwein MD. 2018. Biodiversity and socioeconomics in the city: a review of the luxury effect. Biology Letters 14:20180082. DOI: 10.1098/rsbl.2018.0082.

Peer) reviewing PDF | (2019:01:34115:1:0:NEW 19 Mar 2019) 
397

398

399

400

401

402

403

404

405

406

407

408

409

410

411

412

413

414

415

416

417

418

419

420

421

422

423

424

425

426

427

428

429

430

431

432

433

434

435

436

437

438

439

440

Leong M, Ponisio LC, Kremen C, Thorp RW, Roderick GK. 2016. Temporal dynamics influenced by global change: bee community phenology in urban, agricultural, and natural landscapes. Global Change Biology 22:1046-1053. DOI: 10.1111/gcb.13141.

Lister BC, Garcia A. 2018. Climate-driven declines in arthropod abundance restructure a rainforest food web. Proceedings of the National Academy of Sciences 115:E10397E10406. DOI: 10.1073/pnas.1722477115.

McDonnell MJ, Hahs AK. 2008. The use of gradient analysis studies in advancing our understanding of the ecology of urbanizing landscapes: current status and future directions. Landscape Ecology 23:1143-1155. DOI: 10.1007/s10980-008-9253-4.

McKinney ML. 2006. Urbanization as a major cause of biotic homogenization. Biological Conservation 127:247-260. DOI: 10.1016/j.biocon.2005.09.005.

McKinney ML. 2008. Effects of urbanization on species richness: A review of plants and animals. Urban Ecosystems 11:161-176. DOI: 10.1007/s11252-007-0045-4.

Mimet A, Pellissier V, Quénol H, Aguejdad R, Dubreuil V, Rozé F. 2009. Urbanisation induces early flowering: evidence from Platanus acerifolia and Prunus cerasus. International Journal of Biometeorology 53:287-298. DOI: 10.1007/s00484-009-0214-7.

Nuzzo V. 1999. Invasion Pattern of Herb Garlic Mustard (Alliaria petiolata) in High Quality Forests. Biological Invasions 1:169-179. DOI: 10.1023/A:1010009514048.

Oksanen J, Blanchet FG, Kindt R, Legendre P, Minchin PR, O’Hara RB, Simpson GL, Solymos P, Stevens MHH, Wagner H. 2015. vegan: Community Ecology Package.

Pearse WD, Cavender-Bares J, Hobbie SE, Avolio ML, Bettez N, Roy Chowdhury R, Darling LE, Groffman PM, Grove JM, Hall SJ, Heffernan JB, Learned J, Neill C, Nelson KC, Pataki DE, Ruddell BL, Steele MK, Trammell TLE. 2018. Homogenization of plant diversity, composition, and structure in North American urban yards. Ecosphere 9:n/an/a. DOI: $10.1002 /$ ecs2.2105.

Quinn GP, Keough MJ. 2002. Multidimensional scaling and cluster analysis. In: Experimental Design and Data Analysis for Biologists. Cambridge University Press, 478.

Rebele F. 1994. Urban Ecology and Special Features of Urban Ecosystems. Global Ecology and Biogeography Letters 4:173-187. DOI: 10.2307/2997649.

Savard J-PL, Clergeau P, Mennechez G. 2000. Biodiversity concepts and urban ecosystems. Landscape and Urban Planning 48:131-142. DOI: 10.1016/S0169-2046(00)00037-2.

Schuttler SG, Sorensen AE, Jordan RC, Cooper C, Shwartz A. 2018. Bridging the nature gap: can citizen science reverse the extinction of experience? Frontiers in Ecology and the Environment 16:405-411. DOI: 10.1002/fee.1826.

Schwartz MW, Thorne JH, Viers JH. 2006. Biotic homogenization of the California flora in urban and urbanizing regions. Biological Conservation 127:282-291. DOI: 10.1016/j.biocon.2005.05.017.

Socolar JB, Gilroy JJ, Kunin WE, Edwards DP. 2016. How Should Beta-Diversity Inform Biodiversity Conservation? Trends in Ecology \& Evolution 31:67-80. DOI: 10.1016/j.tree.2015.11.005.

La Sorte FA, McKinney ML, Pyšek P. 2007. Compositional similarity among urban floras within and across continents: biogeographical consequences of human-mediated biotic interchange. Global Change Biology 13:913-921. DOI: 10.1111/j.13652486.2007.01329.x.

Peer] reviewing PDF | (2019:01:34115:1:0:NEW 19 Mar 2019) 
441 Spear DM, Pauly GB, Kaiser K. 2017. Citizen Science as a Tool for Augmenting Museum

442

443

444

445

446

447

448

449

450

451

452

453

454

455

456

457

458

459

460

461
Collection Data from Urban Areas. Frontiers in Ecology and Evolution 5. DOI: 10.3389/fevo.2017.00086.

Stinson KA, Campbell SA, Powell JR, Wolfe BE, Callaway RM, Thelen GC, Hallett SG, Prati D, Klironomos JN. 2006. Invasive Plant Suppresses the Growth of Native Tree Seedlings by Disrupting Belowground Mutualisms. PLOS Biology 4:e140. DOI: 10.1371/journal.pbio.0040140.

Sushinsky JR, Rhodes JR, Possingham HP, Gill TK, Fuller RA. 2013. How should we grow cities to minimize their biodiversity impacts? Global Change Biology 19:401-410. DOI: $10.1111 / \mathrm{gcb} .12055$.

Tryjanowski P, Sparks TH, Kuźniak S, Czechowski P, Jerzak L. 2013. Bird Migration Advances More Strongly in Urban Environments. PLOS ONE 8:e63482. DOI: 10.1371/journal.pone.0063482.

Tsutsui ND, Suarez AV, Holway DA, Case TJ. 2000. Reduced genetic variation and the success of an invasive species. Proceedings of the National Academy of Sciences 97:5948-5953. DOI: $10.1073 /$ pnas.100110397.

Williams NSG, Schwartz MW, Vesk PA, McCarthy MA, Hahs AK, Clemants SE, Corlett RT, Duncan RP, Norton BA, Thompson K, McDonnell MJ. 2009. A conceptual framework for predicting the effects of urban environments on floras. Journal of Ecology 97:4-9. DOI: $10.1111 / \mathrm{j} .1365-2745.2008 .01460 . x$. 


\section{Figure 1}

Map of included City Nature Challenge cities.

The 14 cities are color grouped into five major regions: East, Midwest, South, Southwest, and West. The size of the circle markers represent the relative number of observations coming from each city. Miami had the fewest observations $(1,011)$ and the San Francisco Bay Area had the most $(15,733)$. The average number of observations of the 14 cities was 5,077 $+/-3,817$.

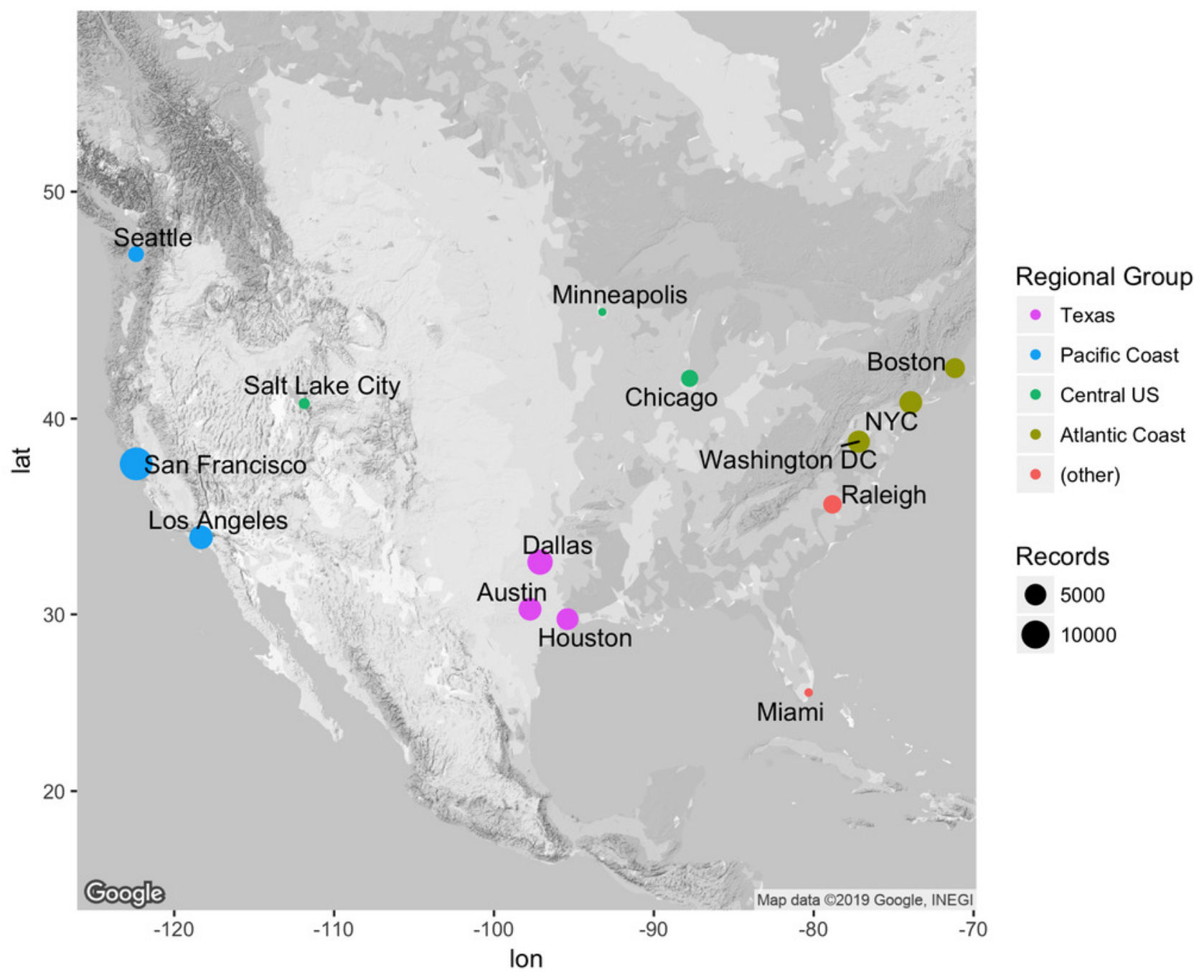


Figure 2

Community composition NMDS plots with all taxa included.

Built from a Bray-Curtis dissimilarity matrix, each point represents the community

composition of a unique combination of one of the five urbanization intensity levels in one of the 14 cities. NMDS 2-D stress $=0.176$. The two plots below are the same except different grouping visualizations are emphasized: in (A) points are grouped together by land cover type; in (B) points are grouped together based on city.

A

\section{B}

\section{All taxa}

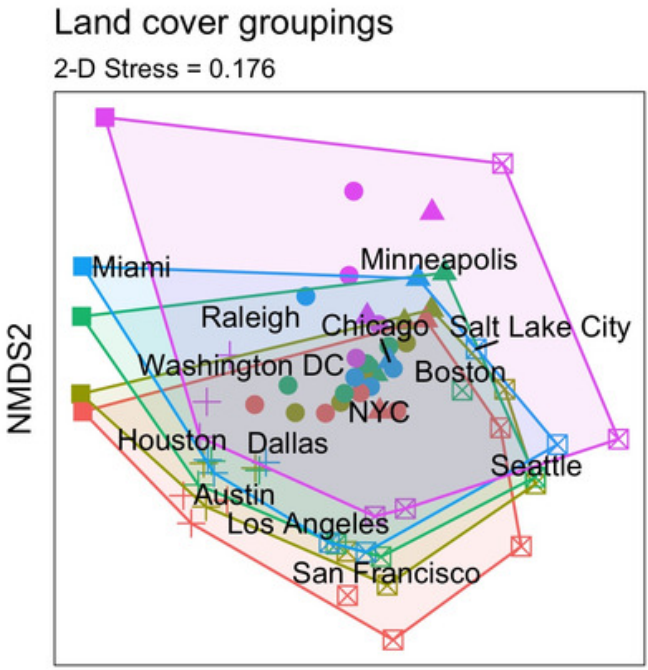

NMDS1

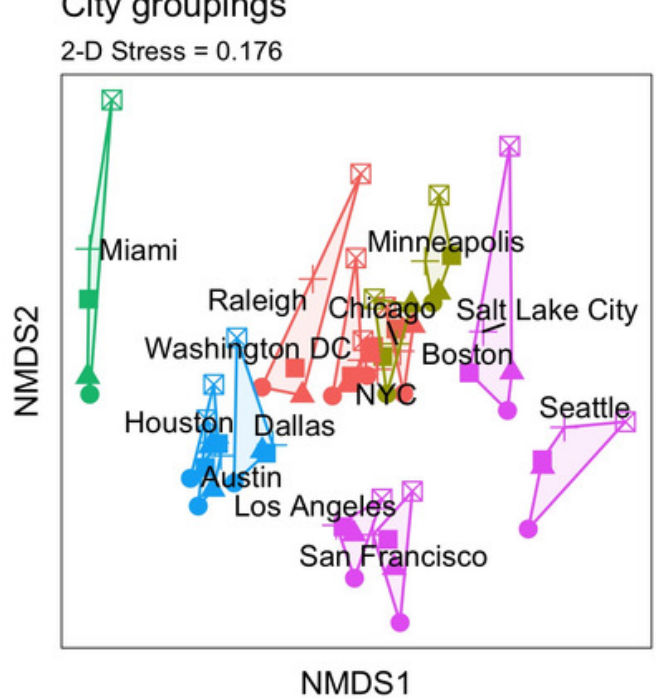

Regions

- East

A Midwest

- South

+ Southwest

$\otimes$ West

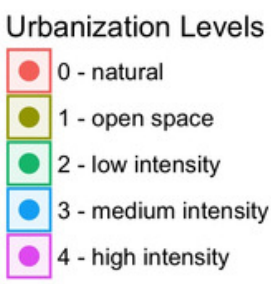

Regions

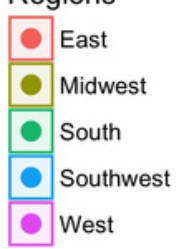

Urbanization Levels

- 0 -natural

A 1 - open space

- 2 - low intensity

+3 -medium intensity

\4-high intensity 


\section{Figure 3}

Community composition NMDS plots for each regional triad with all taxa included.

Built from Bray-Curtis dissimilarity matrices, each plot represents the community composition of a unique combination of one of the five urbanization intensity levels for one of the 3 focal cities for each region. Plots are in order of increasing geographic distance between cities (Texan cities are $\sim 300 \mathrm{~km}$ apart, whereas the Central US cities are $\sim 1500 \mathrm{~km}$ apart), and are grouped to highlight land cover type. (A) Texas (Austin, Dallas, and Houston); NMDS 2-D stress $=0.111$. (B) Atlantic Coast (Boston, New York City, and Washington DC); NMDS 2-D stress $=0.0887$. (C) Pacific Coast (Los Angeles, San Francisco, and Seattle); NMDS 2-D stress $=0.0367$. (D) Central US (Chicago, Minneapolis, and Salt Lake City); NMDS 2-D stress $=0.0664$. 
A

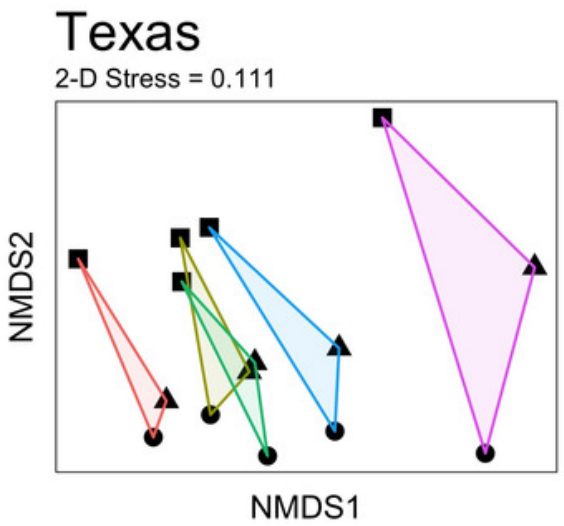

C

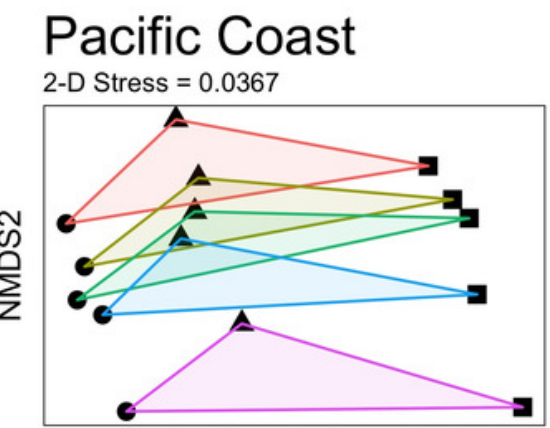

NMDS1

\section{Cities}

- Austin

$\Delta$ Dallas

- Houston

Urbanization Levels
\begin{tabular}{|l|l}
$\square$ & 0 - natural \\
\hline$\square$ & 1 - open space \\
$\square$ & 2 - low intensity \\
\hline$\square$ & 3 - medium intensity \\
\hline$\square$ & 4 - high intensity
\end{tabular}

\section{Cities}

- Los Angeles

A San Francisco

- Seattle

Urbanization Levels

0 - natural

1 - open space

2 - low intensity

3 - medium intensity

4 - high intensity

\section{All taxa}

B

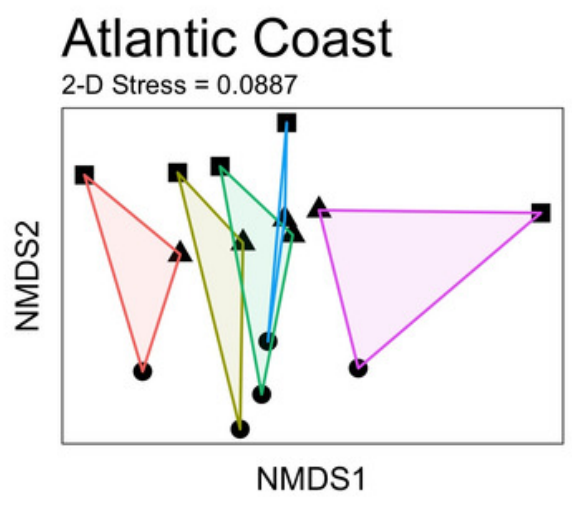

Cities

- Boston

$\Delta$ NYC

- Washington DC

Urbanization Levels

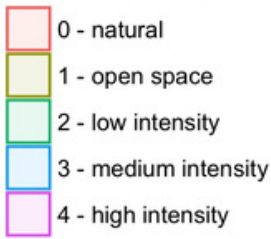

D

Central US

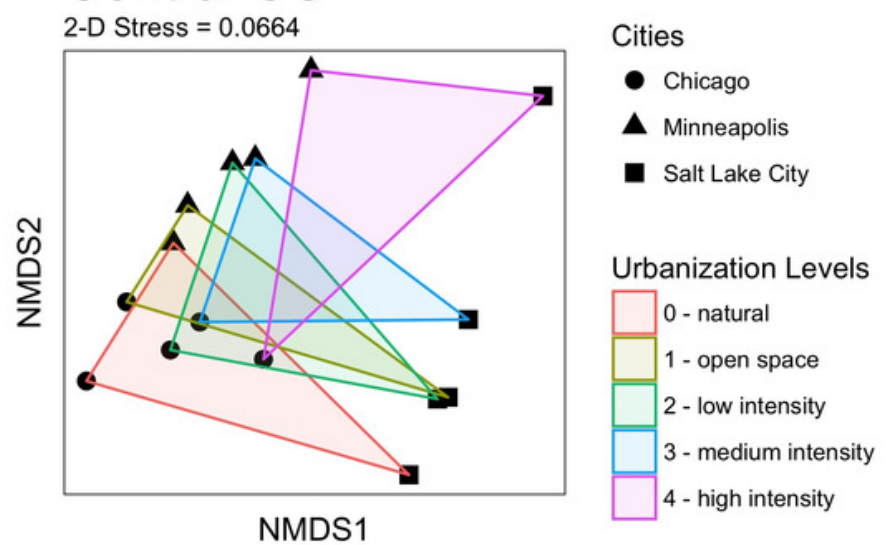




\section{Table 1 (on next page)}

Urban land cover definitions table.

Descriptions of urbanization are based on MRLC'S NLCD2011 definitions (https://www.mrlc.gov/nlcd11_leg.php). 
1 Table 1. Urban land cover definitions table. Descriptions of urbanization are based on 2 MRLC's NLCD2011 definitions (https://www.mrlc.gov/nlcd11_leg.php).

3

4

\begin{tabular}{|l|l|l|}
\hline Code & Land Cover Type & Description \\
\hline $\mathrm{n}$ & natural & all areas not classified as developed, agricultural, or water \\
\hline d1 & $\begin{array}{l}\text { developed - open } \\
\text { space }\end{array}$ & $\begin{array}{l}\text { areas with a mixture of some constructed materials, but } \\
\text { mostly vegetation in the form of lawn grasses. Impervious } \\
\text { surfaces account for less than 20\% of total cover. These } \\
\text { areas most commonly include large-lot single-family } \\
\text { housing units, parks, golf courses, and vegetation planted } \\
\text { in developed settings for recreation, erosion control, or } \\
\text { aesthetic purposes. }\end{array}$ \\
\hline d2 & $\begin{array}{l}\text { developed - low } \\
\text { intensity }\end{array}$ & $\begin{array}{l}\text { areas with a mixture of constructed materials and } \\
\text { vegetation. Impervious surfaces account for 20\% to 49\% } \\
\text { percent of total cover. These areas most commonly } \\
\text { include single-family housing units. }\end{array}$ \\
\hline d3 & $\begin{array}{l}\text { developed - } \\
\text { medium intensity }\end{array}$ & $\begin{array}{l}\text { areas with a mixture of constructed materials and } \\
\text { vegetation. Impervious surfaces account for 50\% to 79\% of } \\
\text { the total cover. These areas most commonly include } \\
\text { single-family housing units. }\end{array}$ \\
\hline d4 & $\begin{array}{l}\text { highly developed areas where people reside or work in } \\
\text { intensity } \\
\text { row houmbers. Examples include apartment complexes, } \\
\text { surfaces account for } 80 \% \text { to 100\% of the total cover. }\end{array}$ \\
\hline
\end{tabular}

5 
Table 2 (on next page)

Taxa-based counts of species found in the majority of cities. 
1 Table 2. Taxa-based counts of species found in the majority of cities.

2

\begin{tabular}{|c|c|c|c|c|c|c|}
\hline & \multicolumn{2}{|c|}{ Cosmopolitan Pool } & \multicolumn{2}{|l|}{ Total Pool } & \multicolumn{2}{|c|}{ Proportion Cosmopolitan } \\
\hline Taxon & num species & observations & num species & observations & num species & observations \\
\hline amphibians & 1 & 81 & 58 & 725 & $1.72 \%$ & $11.17 \%$ \\
\hline birds & 36 & 5258 & 355 & 8115 & $10.14 \%$ & $64.79 \%$ \\
\hline conifers & 1 & 124 & 45 & 786 & $2.22 \%$ & $15.78 \%$ \\
\hline dicots & 36 & 5696 & 2380 & 37744 & $1.51 \%$ & $15.09 \%$ \\
\hline ferns & 0 & 0 & 57 & 869 & $0.00 \%$ & $0.00 \%$ \\
\hline gastropods & 0 & 0 & 113 & 719 & $0.00 \%$ & $0.00 \%$ \\
\hline insects & 7 & 1283 & 835 & 5067 & $0.84 \%$ & $25.32 \%$ \\
\hline mammals & 7 & 938 & 66 & 1698 & $10.61 \%$ & $55.24 \%$ \\
\hline monocots & 1 & 33 & 499 & 5527 & $0.20 \%$ & $0.60 \%$ \\
\hline reptiles & 4 & 334 & 137 & 2123 & $2.92 \%$ & $15.73 \%$ \\
\hline other & 7 & 430 & 664 & 2836 & $1.05 \%$ & $15.16 \%$ \\
\hline TOTALS & 100 & 14177 & 5209 & 66209 & $1.92 \%$ & $21.41 \%$ \\
\hline
\end{tabular}

3 


\section{Table 3 (on next page)}

PERMANOVA results.

We subdivided observations based on their land cover type, then looked for the effect of regional location. For each subset of observations, we buit built Bray-Curtis dissimilarity matrices then conducted PERMANOVA (Permutational Multivariate Analysis of Variance) analyses with 999 iterations. We repeated this for the entire dataset, plants only, and animals only. 
1 Table 3. PERMANOVA results. We subdivided observations based on their land cover type, 2 then looked for the effect of regional location. For each subset of observations, we built built 3 Bray-Curtis dissimilarity matrices then conducted PERMANOVA (Permutational Multivariate 4 Analysis of Variance) analyses with 999 iterations. We repeated this for the entire dataset, plants 5 only, and animals only.

\begin{tabular}{|l|l|r|r|r|}
\hline Taxon & Urban Intensity & \multicolumn{2}{l}{$\mathbf{R}^{\mathbf{2}}$} & \multicolumn{1}{l}{-value } \\
\hline all & natural & 0.486 & 0.001 & 24.494 \\
\hline all & developed - open space & 0.496 & 0.001 & 23.783 \\
\hline all & developed - low intensity & 0.471 & 0.001 & 24.048 \\
\hline all & developed - medium intensity & 0.454 & 0.001 & 24.304 \\
\hline all & developed - high intensity & 0.400 & 0.001 & 26.237 \\
\hline plants & natural & 0.488 & 0.001 & 24.977 \\
\hline plants & developed - open space & 0.501 & 0.001 & 24.477 \\
\hline plants & developed - low intensity & 0.472 & 0.001 & 25.168 \\
\hline plants & developed - medium intensity & 0.452 & 0.001 & 25.601 \\
\hline plants & developed - high intensity & 0.406 & 0.003 & 27.424 \\
\hline animals & natural & 0.490 & 0.001 & 22.597 \\
\hline animals & developed - open space & 0.480 & 0.001 & 22.161 \\
\hline animals & developed - low intensity & 0.469 & 0.001 & 22.034 \\
\hline animals & developed - medium intensity & 0.463 & 0.001 & 22.040 \\
\hline animals & developed - high intensity & 0.393 & 0.010 & 24.728 \\
\hline
\end{tabular}

